\title{
Coriolis Effect on Dynamic Stall in a Vertical Axis Wind Turbine at Moderate Reynolds Number
}

\author{
Hsieh-Chen Tsai* and Tim Colonius ${ }^{\dagger}$ \\ California Institute of Technology, Pasadena, CA 91125, USA
}

\begin{abstract}
The immersed boundary method is used to simulate the flow around a two-dimensional rotating NACA 0018 airfoil at sub-scale Reynolds number in order to investigate the separated flow occurring on a vertical-axis wind turbine. The influence of dynamic stall on the forces is characterized as a function of tip-speed ratio. The influence of the Coriolis effect is also investigated by comparing the rotating airfoil to one undergoing a equivalent planar motion, which is composed of surging and pitching motions that produce an equivalent speed and angle-of-attack variation over the cycle. When the angle of attack of a rotating airfoil starts to decrease in the upwind half cycle, the Coriolis force leads to a wake-capturing phenomenon of a vortex pair at low tip-speed ratio. This effects occurs at a slightly different phase in each cycle and leads to a significant decrease in the average lift during the downstroke phase. Moreover, the wake-capturing is only observed when the combination of surging, pitching, and Coriolis force are present. Finally, an actuator model is placed at an appropriate location on the suction side of the airfoil surface to control the wake-capturing phenomenon. Based on preliminary simulations, a momentum coefficient above 0.02 was able to increase the average lift by more than $70 \%$ over the upwind-half
\end{abstract} cycle.

\section{Nomenclature}

airfoil chord length

lift coefficient, $\left(\frac{L}{\frac{1}{2} \rho W_{\text {inst }}^{2} c}\right)$ momentum coefficient of the actuator, $\left(\frac{\bar{u}_{\text {jet }}^{2} \Delta x}{\frac{1}{2} U_{\infty}^{2} c}\right)$

reduced frequency, $\left(\Omega c /\left(2 U_{\infty}\right)=\lambda /(2 \ell)\right)$

pressure

radius of the turbine

Reynolds Number, $\left(U_{\infty} c / \nu\right)$

Rossby Number, $\left(U_{\infty} /(2 \Omega c)=\ell /(2 \lambda)\right)$

velocity of the fluid in the rotating frame of reference

velocity introduced by the change of variables

average velocity of the jet injected by the actuator

freestream velocity

incoming velocity

position vector in the rotating frame of reference

angle of attack

pitch rate

spatial distribution of the bodyforce actuation

tip-speed ratio

fluid kinematic viscosity

fluid density

*Graduate Student, Mechanical Engineering, Student Member AIAA

${ }^{\dagger}$ Professor, Mechanical Engineering, Associate Fellow AIAA 


$\begin{array}{ll}\theta & \text { azimuthal angle } \\ \hat{\omega} & \text { vorticity of the fluid in the rotating frame of reference } \\ \omega^{\prime} & \text { vorticity introduced by the change of variables } \\ \Delta t & \text { time step } \\ \Delta x & \text { grid spacing } \\ \Omega & \text { angular velocity of the turbine } \\ \ell & \text { ratio of the radius of the turbine to the chord length, }(R / c)\end{array}$

\section{Subscripts}

$\begin{array}{ll}\text { avg } & \text { average velocity } \\ \text { inst } & \text { instantaneous velocity } \\ \text { max } & \text { maximum } \\ \text { sin } & \text { sinusoidal variation } \\ \text { surge } & \text { surge velocity } \\ \text { EPM } & \text { the equivalent planar motion } \\ \text { SPM } & \text { the sinusoidal pitching motion } \\ \text { SSPM } & \text { the sinusoidal surging-pitching motion } \\ \text { VAWT } & \text { vertical axis wind turbine }\end{array}$

\section{Introduction}

Vertical axis wind turbines (VAWT) offer several advantages over horizontal axis wind turbines (HAWT), namely: their low sound emission (consequence of their operation at lower tip speed ratios), their insensitivity to yaw wind direction (because they are omnidirectional), and their increased power output in skewed flow. ${ }^{1,2}$ Dabiri et al. ${ }^{3,4}$ investigated the use of counter-rotating VAWTs in order to achieve higher power output per unit land area and smaller wind velocity recovery distance than existing wind farms consisting of HAWTs. In the present study, a two-dimensional VAWT is investigated numerically at sub-scale Reynolds number in order to understand its aerodynamics for the purpose of improving its efficiency and lift generation.

Dynamic stall refers to the delay in the stall of airfoils that are rapidly pitched beyond the static stall angle. It is associated with a substantially higher lift than is obtained quasi-statically. Due to the large variation in angle of attack, dynamic stall occurs on VAWT operating at low tip speed ratios. ${ }^{5}$ Furthermore, the pinch off of the dynamic stall vortex (leading-edge vortex, LEV) causes a sudden decrease in the lift, a rapid increase in the drag, and a nose-down pitching moment. ${ }^{6,7}$

We focus here on the Coriolis effect on dynamic stall in a VAWT in present study. The influence of the Coriolis effect on the dynamic stall is investigated by comparing the rotating airfoil to one undergoing a equivalent planar motion, which is composed by a surging and pitching motion that produces an equivalent speed and angle-of-attack variation over the cycle. The influence of dynamic stall on forces is characterized as a function of tip-speed ratio. Moreover, inspired by Wang et al.,$^{8}$ airfoils undergoing a sinusoidal pitching motion and a sinusoidal surging-pitching motion are also compared to see if these two motions can be an appropriate model for the VAWT.

Several attempts have been made to model a VAWT at $R e_{c} \sim O\left(10^{5}\right)$, which is appropriate to the urban applications of VAWTs, using Unsteady Reynolds-Average Navier-Stokes (URANS) with different turbulence models by investigating a $2 \mathrm{D}$ airfoil undergoing an effective motion ${ }^{9-11}$ and simulating a multi-bladed 2D VAWT. ${ }^{12,13}$ Wang et al. ${ }^{8}$ focused on investigating the dynamic stall in a VAWT numerically by studying an 2D airfoil undergoing the effective sinusoidal pitching motion. The results have a good agreement with experiments. ${ }^{7,15-17}$ Ferreira et al. ${ }^{14}$ simulated dynamic stall in a section of a VAWT using Detached Eddy Simulation (DES) at $R e_{c}=50,000$ and validated the results by comparing the vorticity in the rotor area with particle image velocimetry (PIV) data. 
Active flow control can be used to improve performance of VAWT. Hwang et al. ${ }^{18}$ applied an individual active blade control system to change the pitch angles of the blades individually according to the incoming wind velocity and its azimuthal angles. They were able to improve the power output by $60 \%$ comparing with one with fixed pitch airfoils. Greenblatt et al. ${ }^{19}$ controlled dynamic stall on a double-bladed VAWT using pulsed dielectric barrier discharge (DBD) plasma actuators in a feed-forward control configuration. Based on choosing appropriately a duty cycle and a range of azimuthal angles of plasma actuation initiation and termination, they was able to achieve a net power increase of more than $10 \%$. Inspired by their work, we perform an open loop control of dynamic stall vortices by placing an actuator on the suction surface.

In order to explore the parametric space in relatively short computational time and have more understanding of the details of the vortex dynamics, flows are simulated at moderate Reynolds number $\left(\operatorname{Re}_{c} \sim O\left(10^{3}\right)\right)$ using the immersed boundary projection method. A major limitation of our present approach is the restriction of flow to a two-dimensional cross section of an otherwise planar turbine geometry. We believe that the results will be qualitatively relevant to three-dimensional flows at higher Re, but precise comparisons await future simulations.

\section{Simulation setup and numerical method}

Figure 1 shows the schematic of a VAWT. With a freestream velocity, $U_{\infty}$, coming from the left, a VAWT with a radius $R$ is rotating at an angular velocity $\Omega$. The chord length of the turbine blade is $c$. In order to systematically investigate the aerodynamics of a VAWT, three dimensionless parameter are introduced:

$$
\begin{aligned}
\text { tip-speed ratio: } & \lambda=\frac{\Omega R}{U_{\infty}}, \\
\text { radius-to-chord-length ratio: } & \ell=\frac{R}{c}, \\
\text { Reynolds number: } & R e=\frac{U_{\infty} c}{\nu}, \\
\text { Rossby number: } & R o=\frac{U_{\infty}}{2 \Omega c}=\frac{\ell}{2 \lambda}=\frac{1}{4 k},
\end{aligned}
$$

where $\nu$ is the kinematic viscosity of the fluid and $k$ is the reduce frequency, $k=\frac{\Omega c}{2 U_{\infty}}=\frac{\lambda}{2 \ell}$.

The instantaneous incoming velocity $W_{\text {inst }}$ and the angle of attack $\alpha$ then can be characterized as a function of the tip speed ratio $\lambda$ and the azimuthal angle $\theta$ :

$$
\begin{aligned}
& \alpha(\lambda, \theta)=\tan ^{-1}\left(\frac{\sin \theta}{\lambda+\cos \theta}\right), \\
& \frac{W_{\text {inst }}(\lambda, \theta)}{U_{\infty}}=\sqrt{1+2 \lambda \cos \theta+\lambda^{2}} .
\end{aligned}
$$

igure 2 shows the angle of attack variation and incoming velocity variation of the VAWT at $\lambda=2$. From equation (5), the maximum angle of attack,

$$
\alpha_{\max }(\lambda)=\tan ^{-1}\left(\frac{1}{\sqrt{\lambda^{2}-1}}\right)
$$

occurs at $\theta=-\cos \left(\frac{1}{\lambda}\right)$.

In order to isolate the Coriolis effect on dynamic stall, a moving airfoil experiencing an equivalent incoming velocity and angle-of-attack variation over a cycle is proposed. This equivalent planar motion (EPM) is composed of a surging motion with a velocity $W_{\text {surge }}$ and a pitching motion around the leading edge with a pitch rate $\dot{\alpha}$. The airfoil is undergoing the EPM in a freestream velocity $W_{\text {avg. }} W_{\text {avg }}, W_{\text {surge }}$, and $\dot{\alpha}$ are 
shown to be

$$
\begin{aligned}
& W_{\text {avg }}(\lambda)=\frac{1}{2 \pi} \int_{0}^{2 \pi} W_{\text {inst }}(\lambda, \theta) d \theta=\frac{U_{\infty}}{2 \pi} \int_{0}^{2 \pi} \sqrt{1+2 \lambda \cos \theta+\lambda^{2}} d \theta \\
& W_{\text {surge }}(\lambda, \theta)=W_{\text {inst }}(\lambda, \theta)-W_{\text {avg }}(\lambda) \\
& \dot{\alpha}(\lambda, \theta)=\frac{1}{2 R o}\left(\frac{1+\lambda \cos \theta}{1+2 \lambda \cos \theta+\lambda^{2}}\right)
\end{aligned}
$$

Moreover, due to the periodic oscillation of angle of attack and incoming velocity variation, Wang et $a l .{ }^{8}$ studied dynamic stall in a VAWT by investigating an airfoil undergoing a simplified sinusoidal pitching motion. Inspired by their work, sinusoidal variations in the angle of attack and incoming velocity, which are written as a function of the tip speed ratio $\lambda$ and the azimuthal angle $\theta$ in equations (11) and (12) and shown in figure 2, are also considered:

$$
\begin{aligned}
& \alpha_{\sin }(\lambda, \theta)=\alpha_{\max }(\lambda) \sin \theta, \\
& \frac{W_{\mathrm{inst}, \sin }(\lambda, \theta)}{U_{\infty}}=\lambda+\cos \theta .
\end{aligned}
$$

Although the sinusoidal motion shares the same amplitude, it overestimates the angle of attack in the upstroke phase, underestimates the incoming velocity in the downstroke phase, and slightly underestimates the instantaneous velocity over the entire half-cycle. In order to search for the most appropriate model for a VAWT, we introduce two additional motions: a sinusoidal pitching motion (SPM) and a sinusoidal surging-pitching motion (SSPM). Airfoils undergoing both the SPM and SSPM pitch with the sinusoidal angle-of-attack variation described in equation (11) in a freestream velocity $W_{\mathrm{avg}, \sin }=\lambda U_{\infty}$. Airfoils undergoing the SSPM also surge with a velocity $W_{\text {surge }, \sin }=U_{\infty} \cos \theta$.

NACA 0018 airfoils are used as blades in present study. In preliminary simulations of a three-bladed VAWT as well as in previous studies ${ }^{14}$ vorticity-blade interaction is only observed in the downwind-half of a cycle. Because only the flow in the upwind-half cycle is important to torque generation (and to save computational time), we use a single-bladed turbine. The immersed boundary projection method developed by Colonius et al. ${ }^{20}$ is used to compute two-dimensional incompressible flows in an airfoil-fixed reference frame with appropriate forces added to the momentum equation to account for the non-inertial reference frame shown in figure 1. The equations are solved on multiple overlapping and progressively coarser grids. Six grid levels are used for the present computations. Each level has the same number of grid points $(600 \times 600)$, and the coarsest grid extends to 96 chord lengths in both the transverse and streamwise directions. The ratio of the radius of the turbine to the chord length, $\ell$, is chosen to be 6 . The time step, $\Delta t U_{\infty} / c$, is chosen to make the CFL number less than 0.4. In order to remove transients associated with the startup of periodic motion, it was deemed sufficient to compute about five periods of motion. An additional five periods of stationary-state nearly periodic motion were then computed.

As noted above, we solve the dimensionless momentum equation in the rotating frame of reference

$$
\frac{\partial \hat{\mathbf{u}}}{\partial t}+(\hat{\mathbf{u}} \cdot \nabla) \hat{\mathbf{u}}=-\nabla p+\frac{1}{R e} \nabla^{2} \hat{\mathbf{u}}-\frac{d \boldsymbol{\Omega}}{d t} \times \hat{\mathbf{x}}-2 \boldsymbol{\Omega} \times \hat{\mathbf{u}}-\boldsymbol{\Omega} \times(\boldsymbol{\Omega} \times \hat{\mathbf{x}}),
$$

where $\hat{\mathbf{u}}$ and $\hat{\mathbf{x}}$ are the fluid velocity and the position vector in the rotating frame of reference and $\boldsymbol{\Omega}=\frac{1}{2 R o}$ is the dimensionless angular velocity of rotating frame of reference. We then introduce the change of variables

$$
\begin{aligned}
\mathbf{u}^{\prime} & =\hat{\mathbf{u}}+\boldsymbol{\Omega} \times \hat{\mathbf{x}} \\
\boldsymbol{\omega}^{\prime} & =\hat{\boldsymbol{\omega}}+2 \boldsymbol{\Omega},
\end{aligned}
$$

where $\hat{\boldsymbol{\omega}}=\nabla \times \hat{\mathbf{u}}$ is the vorticity field in the rotating frame of reference. By taking divergence and curl of equation (13), we will have the following vorticity and pressure equation

$$
\begin{aligned}
& \frac{\partial \boldsymbol{\omega}^{\prime}}{\partial t}=\nabla \times\left(\hat{\mathbf{u}} \times \boldsymbol{\omega}^{\prime}\right)-\frac{1}{R e} \nabla \times\left(\nabla \times \boldsymbol{\omega}^{\prime}\right) \\
& \nabla^{2}\left(p+\frac{1}{2}|\hat{\mathbf{u}}|^{2}-\frac{1}{2}|\boldsymbol{\Omega} \times \hat{\mathbf{x}}|^{2}\right)=\nabla \cdot\left(\hat{\mathbf{u}} \times \boldsymbol{\omega}^{\prime}\right)
\end{aligned}
$$




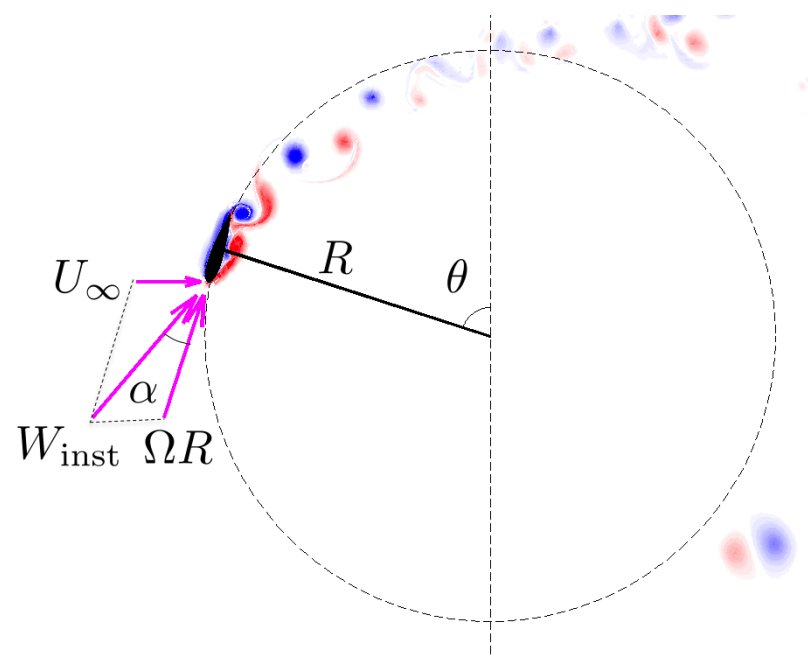

Figure 1. Schematic of a VAWT and the computational domain.

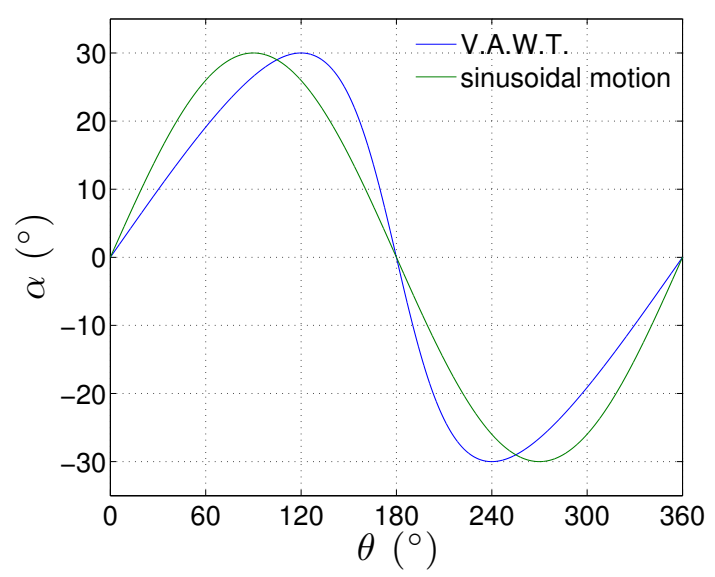

(a) Angle of attack variation

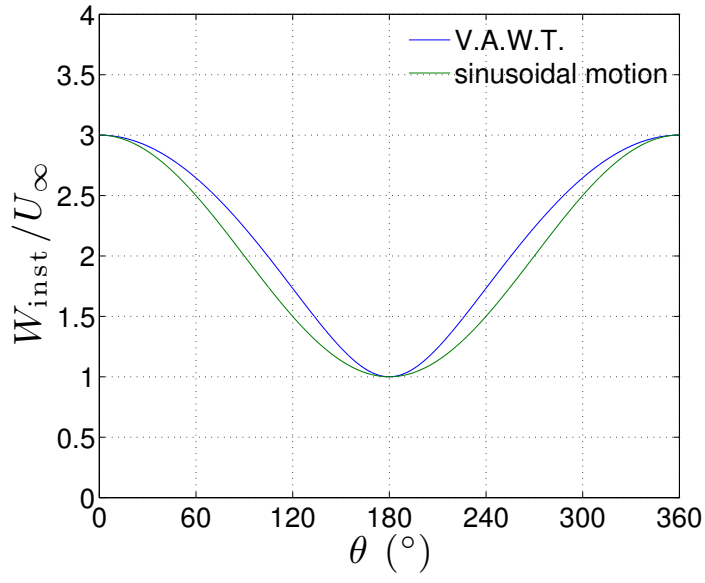

(b) Incoming velocity variation

Figure 2. Comparison of angle of attack variation and incoming velocity variation between the VAWT and the sinusoidal motion at $\lambda=2$. 
Since the flow is incompressible and two-dimensional, the first term in the right hand side of equation (16) is just the advection of vorticity with velocity $\hat{\mathbf{u}}$. Therefore in the body-fixed frame of reference, Coriolis force does not generate vorticity except on the boundary so that it only changes the way vorticity propagates in free space. In the surging-pitching configuration shown in figures 4, since the frame of reference is rotating clockwise, the Coriolis force deflects the fluid in the clockwise direction. Moreover, because the magnitude of Coriolis force is proportional to the magnitude of velocity, fluid with high velocity will be deflected more rapidly. The dynamic of vortices could be affected dramatically by Coriolis force because of relatively high fluid local velocity compared to the surrounding values.

\section{Results}

\section{A. Qualitative flow features in a VAWT}

We begin with examining flows at low tip-speed ratio, $\lambda=2, R o=1.5$, and $R e=1500$, which gives a maximum amplitude of 30 degrees in angle of attack variation and a reduced frequency $k=1 / 6$. Figure 3 shows the vorticity field generated by the blade at different azimuthal angles over a cycle. Negative and positive vorticity are plotted in blue and red contour levels, respectively, and all vorticity contour plots are using the same contour levels.

At the beginning of a cycle, as shown in figure 3(a), the airfoils just are just returning to zero angle of attack, and there are still the remnants of earlier vortex shedding in the wake. The flow reattaches by the angle depicted in figure 3(b). When the angle of attack increases further, the wake behind the airfoil starts to oscillate and vortex shedding commences. We can see the key stages of dynamic stall including growth, pinch-off and advection of an LEV on the suction side in figures 3(c-e). The vortices generated will propagate downstream into the wake of the VAWT or interact with the blades in the downwind half of a cycle.

When the angle of attack starts to decrease, as shown in figure 3(f), a TEV develops and we observe Bloor instability occurs in the trailing-edge shear layer at this Reynolds number. This TEV couples with a LEV forming a vortex pair that travels downstream together with the airfoil as shown in figures $3(\mathrm{~g}-\mathrm{i})$. This vortex pair interacts with the airfoil in the downwind half of a cycle as shown in figures $3(\mathrm{j}-\mathrm{l})$, which was also observed by Ferreira et al. ${ }^{14}$

When the blade rotates further, the angle of attack becomes negative. Vortices are now generated on the other side of the airfoil as shown in figures $3(\mathrm{~m}-\mathrm{p})$, and sheds into the wake of the VAWT. For a multi-bladed VAWT, when a blade is traveling in the downwind half of a cycle, it will interact frequently with the vortices generated upstream from the other blades or even itself. We can observe this trend in our single-bladed VAWT simulation as shown in figure 3(o).

\section{B. Comparison of VAWT and EPM}

In this section, we compare flows around an airfoil undergoing the EPM and a single-bladed VAWT at $\lambda=2$, $R o=1.5$, and $R e=1500$. We are interested in the tangential force response of the blade over a cycle because the power output is proportional to the tangential force acting on the blade when VAWTs operate at a constant tip-speed ratio. From preliminary simulations, a three-bladed VAWT with $\ell=4$ will be free-spinning with a time-averaged tip-speed ratio $\lambda=0.95$ at $R e=1500$ so that in the flow we are examining, the average tangential force is expected to be negative. Nevertheless, since the lift and tangential force have the same trend over a cycle from the preliminary simulations of the free-spinning VAWT, we could alternatively look at the lift response of the blade. Moreover, from previous studies, ${ }^{14}$ due to large vorticity-blade interactions that cancel out the driving torque in the downwind half cycle, the power of a VAWT is generated mostly in the upwind half cycle. Therefore in this study, we will focus on the lift in the upwind-half of a cycle.

In figures 4 , the first two columns show the vorticity field at different azimuthal angles for EPM and VAWT motion. Figure 5 shows the comparisons of the lift coefficients against dimensionless time and angle of attack for a single rotation and for the average of both lift coefficients over five cycles. Although there are still the remnants of earlier vortex shedding in the wake when the airfoil just returns to zero angle of attack (figure 4(a)), the flow reattaches by the angle depicted in figure 4(b), which leads to smoothly increasing lift 


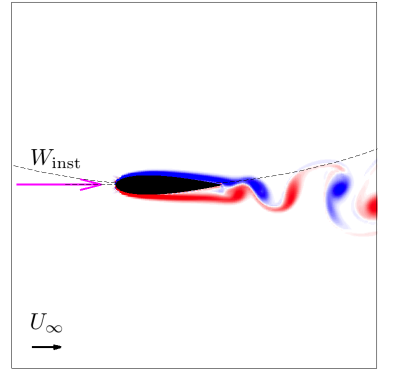

(a) $\theta=0^{\circ} / 360^{\circ}, \alpha=0^{\circ}$.

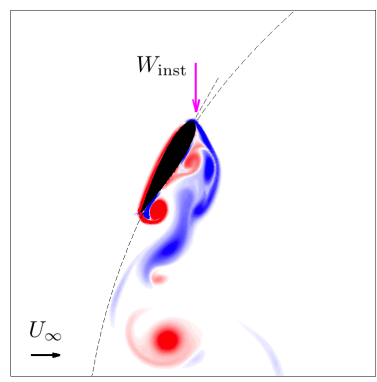

(e) $\theta=120^{\circ}, \alpha=30^{\circ}$.

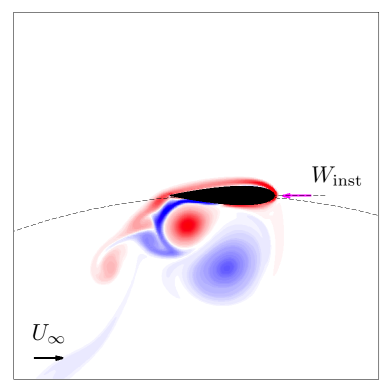

(i) $\theta=180^{\circ}, \alpha=0^{\circ}$.

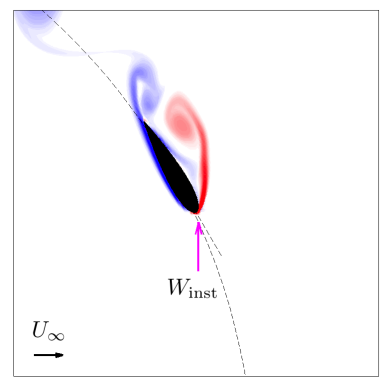

(m) $\theta=240^{\circ}, \alpha=-30^{\circ}$.

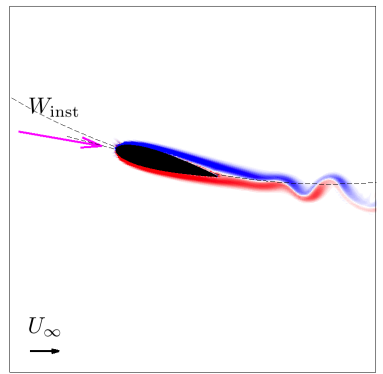

(b) $\theta=15^{\circ}, \alpha=5.0^{\circ}$.

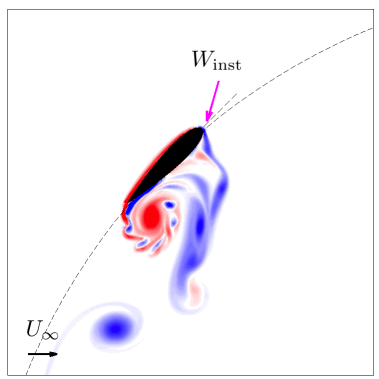

(f) $\theta=135^{\circ}, \alpha=28.7^{\circ}$.

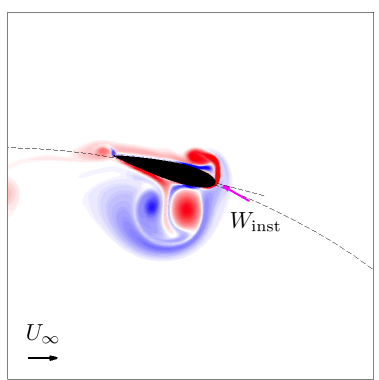

(j) $\theta=195^{\circ}, \alpha=-14.1^{\circ}$.

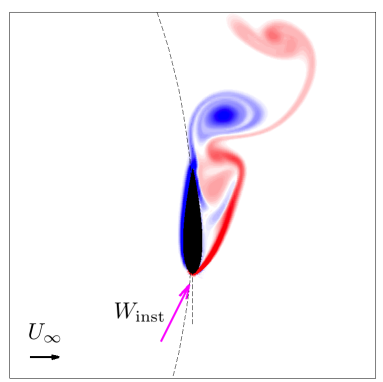

(n) $\theta=270^{\circ}, \alpha=-26.6^{\circ}$.

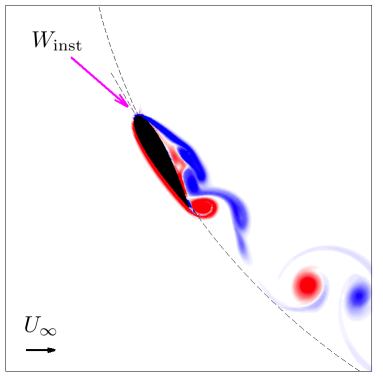

(c) $\theta=60^{\circ}, \alpha=19 \cdot 1^{\circ}$.

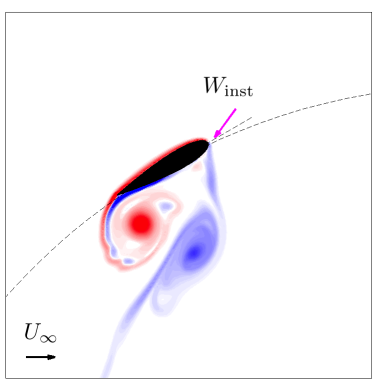

(g) $\theta=150^{\circ}, \alpha=23.8^{\circ}$.

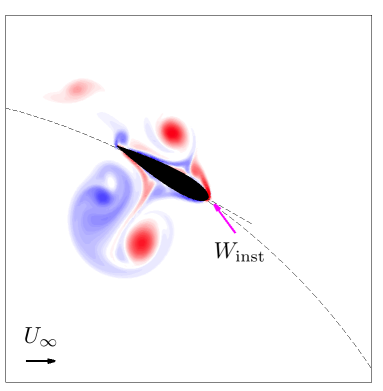

(k) $\theta=210^{\circ}, \alpha=-23.8^{\circ}$.

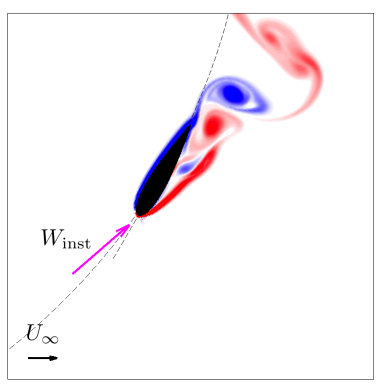

(o) $\theta=300^{\circ}, \alpha=-19.1^{\circ}$.

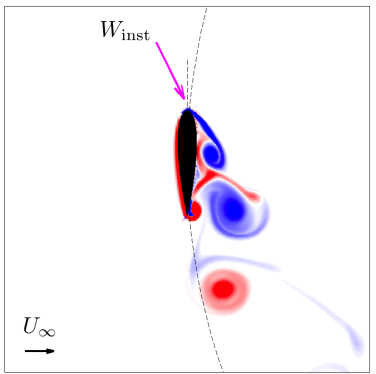

(d) $\theta=90^{\circ}, \alpha=26.6^{\circ}$.

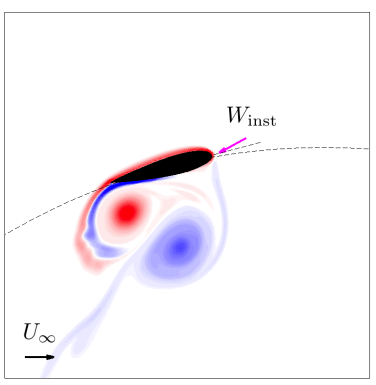

(h) $\theta=165^{\circ}, \alpha=14.1^{\circ}$.

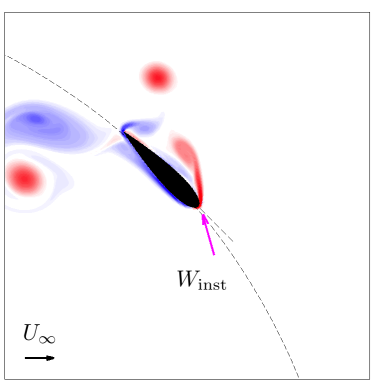

(l) $\theta=225^{\circ}, \alpha=-28.7^{\circ}$.

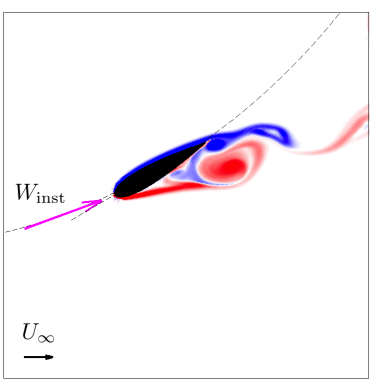

(p) $\theta=330^{\circ}, \alpha=-9.9^{\circ}$.

Figure 3. Vorticity field for a (clockwise rotating) VAWT at various azimuthal angles at $\lambda=2, R o=1.5$, and $R e=1500$. Negative and positive vorticity are plotted in blue and red contour levels, respectively, and all vorticity contour plots are using the same contour levels. 
coefficients at low angle of attack. The differences in lift coefficients between the EPM and VAWT are small as shown in figure 5. As the angle of attack increases, dynamic stall commences as shown in figures 4(c-e), which leads to rapidly increasing lift coefficients. Notice that both EPM and VAWT flows are quite similar with just a small phase difference when the airfoils pitch up; the lift coefficients are similar throughout the upstroke phase.

When the downstroke phase starts (figures 4(e-f)), the development of a TEV leads to a decrease in lift. Bloor instability, ${ }^{21}$ which seems to resemble the convectively unstable Kelvin-Helmholtz instability observed in plane mixing layers, is observed in the shear layer at the trailing edge and produces high frequency fluctuations in the lift coefficient. When the angle of attack decreases further, for EPM, the TEV sheds into the wake and a secondary vortex ${ }^{15}$ appears (figure $4(\mathrm{~g})$ ), which results in a sudden increase in the lift coefficient. On the other hand, for VAWT, as described in section A, this TEV couples with a LEV and forms a vortex pair that travels together with the airfoil (figures $4(\mathrm{~g}-\mathrm{h})$ ). This generates high pressure on the suction side and further decreases the lift. This vortex pair seems to be "captured" by the rotating airfoil. By analogy with flow observed in insect flight by Dickinson et al. ${ }^{22}$ we refer this phenomenon as the wake-capturing of vortex pair in a VAWT. The wake-capturing occurs at a slightly different phase in each cycle and leads to a significant decreases in the average lift in the downstroke phase. In general, the lift of an airfoil undergoing the equivalent motion is overestimated in the downstroke phase. Moreover, when this vortex pair travels downstream, it will interact with the airfoil in the downwind half of a cycle. The lift coefficient will result in large fluctuations and small mean because of this vorticity-blade interaction, which was also observed by Ferreira et al. ${ }^{14}$

We can see from the second and third columns in figure 4 that the Coriolis force deflects the flow around a rotating airfoil in clockwise direction. The magnitude of Coriolis force acting on the background fluid decreases as the azimuthal angle increases. Therefore the Coriolis force acting on the fluid around vortices becomes relatively important in the downstroke phase. From figures $4(\mathrm{f}-\mathrm{h})$, a stronger Coriolis force is exerted on the fluid around the vortex pair, which deflects the fluid in such a way that the vortex pair travels with the airfoil.

\section{Effect of tip-speed ratio, Rossby number, and Reynolds number}

In this section, we examined the flows at tip-speed ratios, $\lambda=2,3$ and 4, and Reynolds number, $R e=500$, 1000 , and 1500 . The corresponding $C_{L \text {,VAWT }}$ and $C_{L, \text { EPM }}$ in flow with with Rossby number, $R o=0.75,1.00$, and 1.25 are shown in figures 6,7 , and 8 , respectively. As tip-speed ratio increases, the amplitudes of angle of attack variation and the corresponding lift decreases. Furthermore, we can see that the large decrease in $C_{L, \text { VAWT }}$ due to wake-capturing of vortex pair occurs in the flows at low tip-speed ratio and higher Reynolds numbers. As Rossby number decreases, which corresponds to a larger Coriolis force, this discrepancy in lift coefficients becomes larger. In preliminary simulations, we have observed this wake-capturing phenomenon for $\lambda=2$ and $R o=1.5$ at an even higher Reynolds number $R e=4000$. Over the range of $\lambda$, Ro, and $R e$ considered here, such a wake-capturing phenomenon could play a role in three-dimensional flows at higher Reynolds number. We can also see that an airfoil undergoing EPM is a good approximation to a rotating airfoil in a VAWT in upstroke phase for various tip-speed ratios, Rossby number, and Reynolds numbers. However, it overestimates the lift coefficients in the downstroke phase in those cases where wake-capturing occurs.

To probe the existence of wake-capturing at high Reynolds number, the vorticity field in VAWT ( $R e=$ 1500) for a single period is compared qualitatively with phase-averaged PIV data from Ferreira et al. ${ }^{14}(R e \approx$ $\left.10^{5}\right)$ at $\lambda=2$ and $R o=1(\ell=4)$. In figure 9(a), Ferreira et al. filter the phase-averaged vorticity field to plot only the LEV generated around $\theta=72^{\circ}$ and overlap its evolutions as the blade travels to various azimuthal angles. Similarly, the filtered phase-averaged TEV evolution is shown in figure $9(\mathrm{c})$. In order to make qualitatively comparison, in figures 9(b) and 9(d), the vorticity fields in VAWT at corresponding azimuthal angles are overlapped to show the evolution of vortices. From figures 9(b) and 9(d), wake-capturing occurs around $\theta=90^{\circ}$, which forms a vortex pair traveling with the blade. The vortex pair then detaches around $\theta=133^{\circ}$ and propagates downstream. The evolution of the phase-averaged LEV in figure 9(a) shows similar behavior. Moreover, at $\theta=158^{\circ}$, the vortex pair composed of the phase-averaged LEV and TEV seems to 


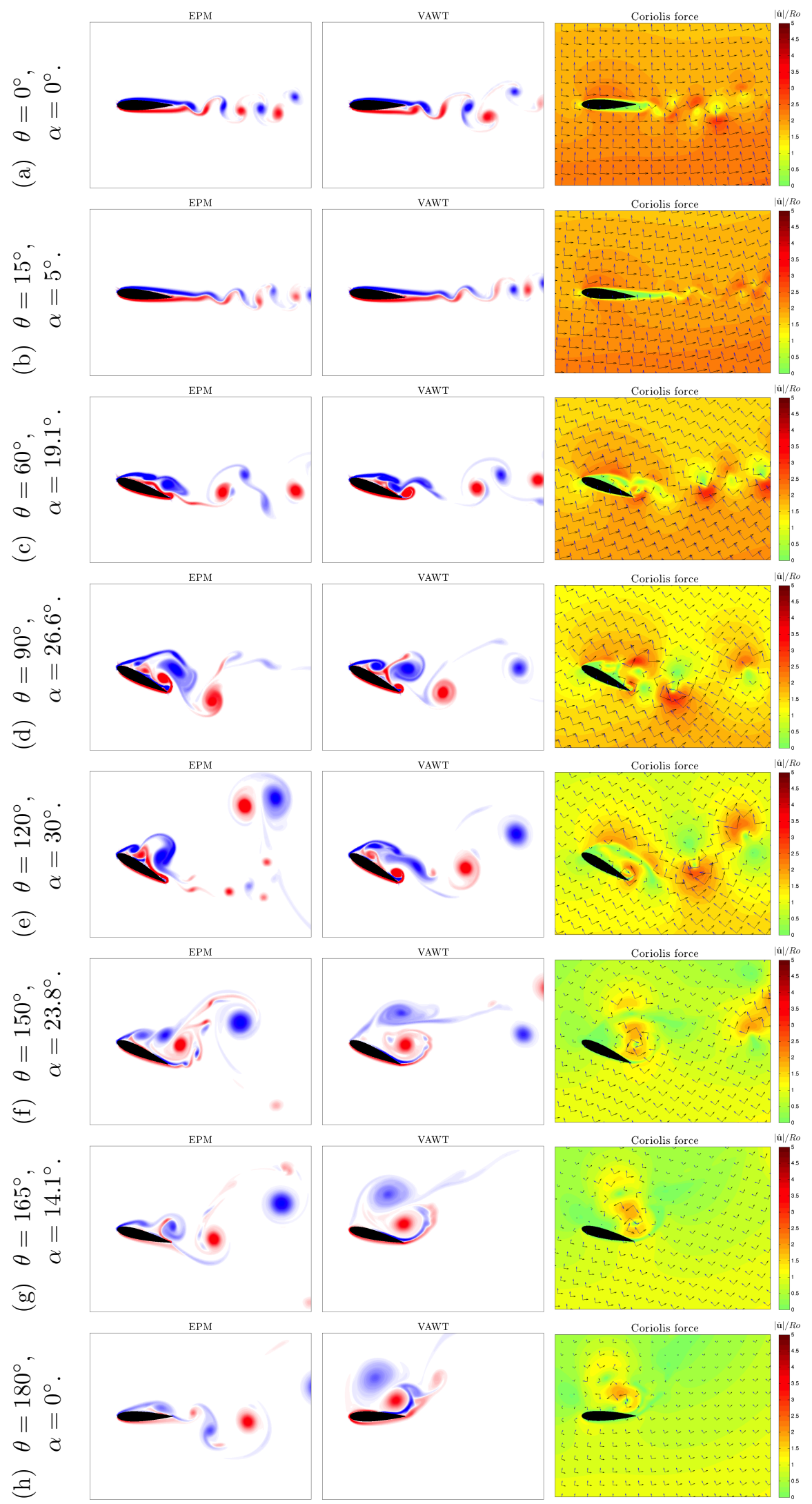

Figure 4. Vorticity field for EPM and VAWT and the Coriolis force for VAWT at various azimuthal angles. Negative and positive vorticity are plotted in blue and red contour levels, respectively, and all vorticity contour plots are using the same contour levels. In the Coriolis force plots, black arrows show the direction of velocity, blue arrows point the direction of the Coriolis force, and the color contour plots the magnitude of the Coriolis force. 


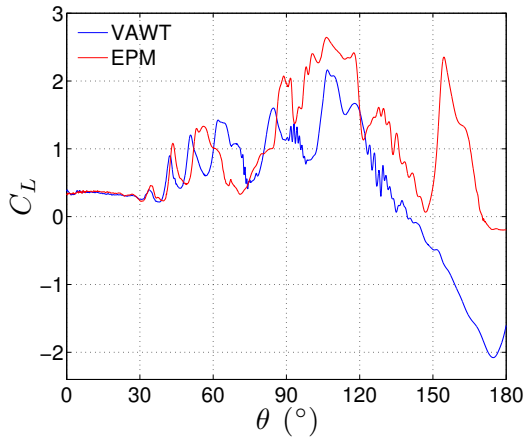

(a) The lift coefficients over a cycle (b) against dimensionless time.

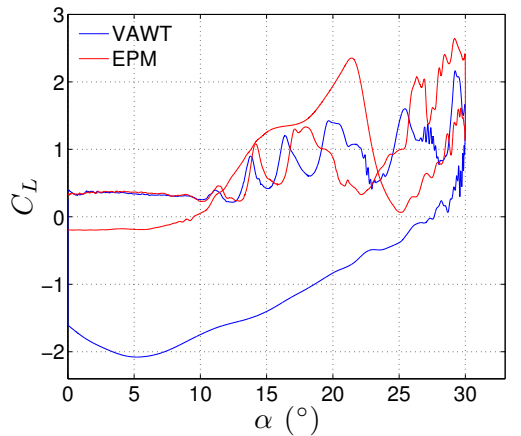

(b) The lift coefficients over a cycle (c) against angle of attack.

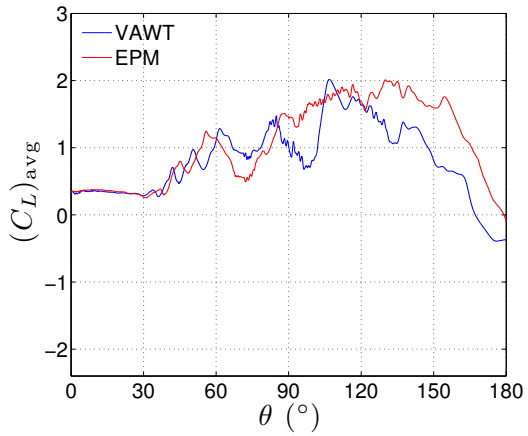

(c) The average lift coefficients over five cycles.

Figure 5. Comparing $C_{L, \mathrm{VAWT}}$ and $C_{L, \mathrm{EPM}}$ at $\lambda=2, R o=1.5$, and $R e=1500$.

be well captured by the simulation. The qualitative agreement suggests that wake-capturing may also be occurring in Ferreira et al.'s experiment.

\section{Decoupling the effect of surging, pitching, and rotation}

The flow around a rotating airfoil in a VAWT is complicated because the angle of attack and incoming velocity vary simultaneously. In order to have more understanding of this flow, we examine independently airfoils undergoing the pitching and surging motion decoupled from EPM and compare their lift responses with one in VAWT.

\section{Airfoil undergoing only surging motion}

We examine a surging motion with fixed angles of attack of 15 and 30 degrees at $\lambda=2, R o=1.5(k=1 / 6)$, and $R e=1500$. A rotating airfoil undergoing only the surging motion in VAWT is achieved by pitching the airfoil around the leading edge simultaneously as it rotates so that the angle of attack is fixed with respect to the incoming velocity. For an airfoil surging at an angle of attack of $15^{\circ}$, lift coefficients are shown in figure 10 (a). We can see that dynamic stall is relatively stable and no wake-capturing phenomenon is observed. Moreover, from the analysis by Choi et al. ${ }^{23}$ when the reduced frequency is low enough, the flow can be approximately quasi-steady, which results in both lift coefficients for VAWT and EPM fluctuating about a slowly increasing mean value. For the case of $\alpha=30^{\circ}$, lift coefficients are shown in figure 10(b). The flow is well separated so that there is no stationary vortex shedding. Moreover, no wake-capturing phenomenon is observed in the flow.

\section{Airfoil undergoing only pitching motion}

We consider a pitching motion in a freestream velocity $W_{\text {avg }}=\lambda U_{\infty}$ at $\lambda=2, R o=1.5$ and $R e=1500$. A rotating airfoil undergoing only the pitching motion in a VAWT is achieved by rotating an airfoil in a VAWT without the external free stream and pitching it around the leading edge with the exact angle of attack variation simultaneously. The corresponding lift coefficients are shown in figure 11 . We can see dynamic stall in both lift coefficients as angle of attack increases. However, there is no wake-capturing.

Table 1 concludes the observations of the wake-capturing phenomenon for all combinations of the motions. No wake-capturing phenomenon is observed in the flow around an airfoil undergoing independent surging motion and pitching motions, nor is it observed for the combined pitching and surging associated with EPM. It seems in this flow to be uniquely associated with the combination of pitch, surge, and Coriolis effect. 


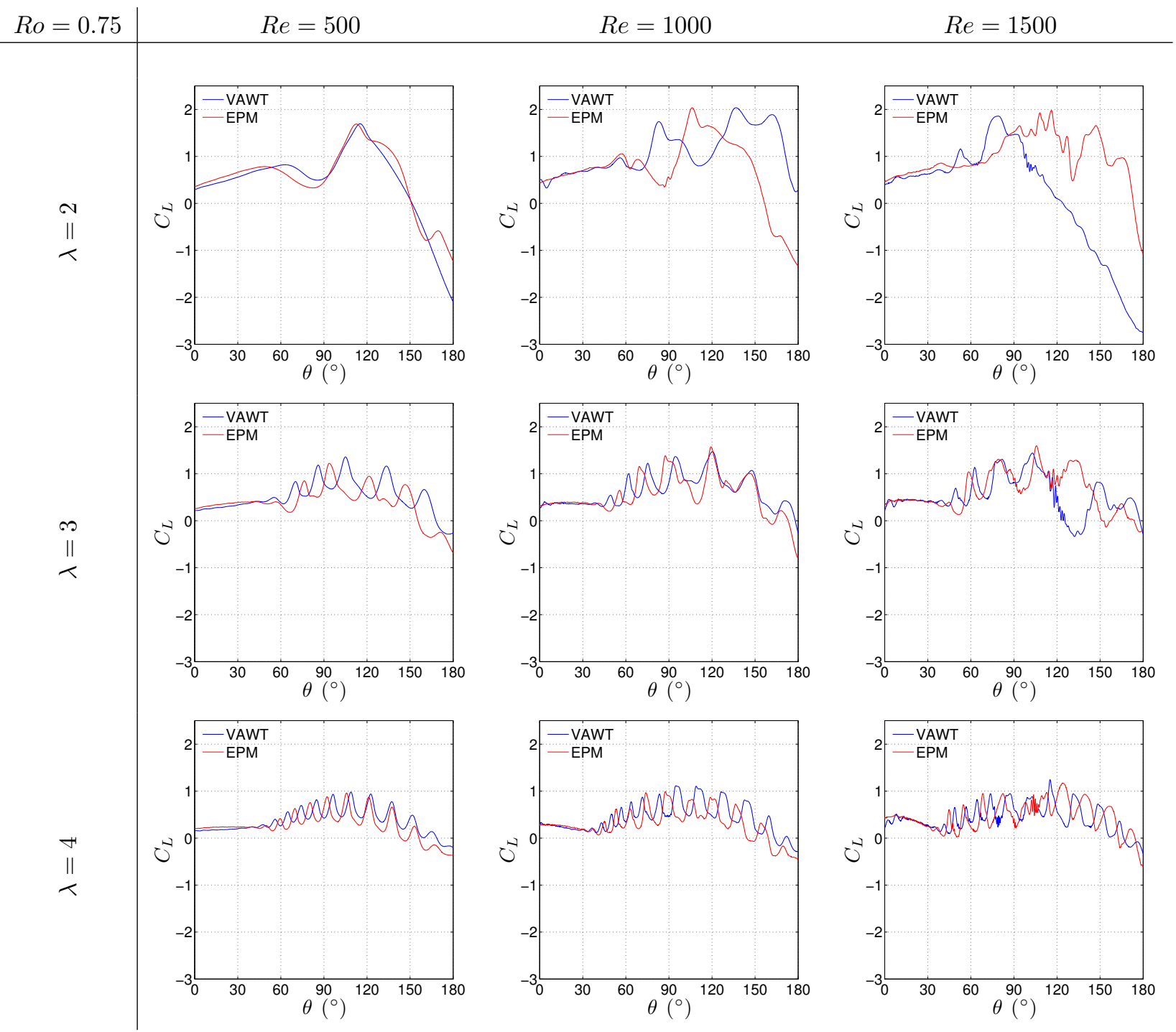

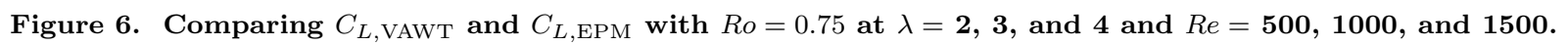




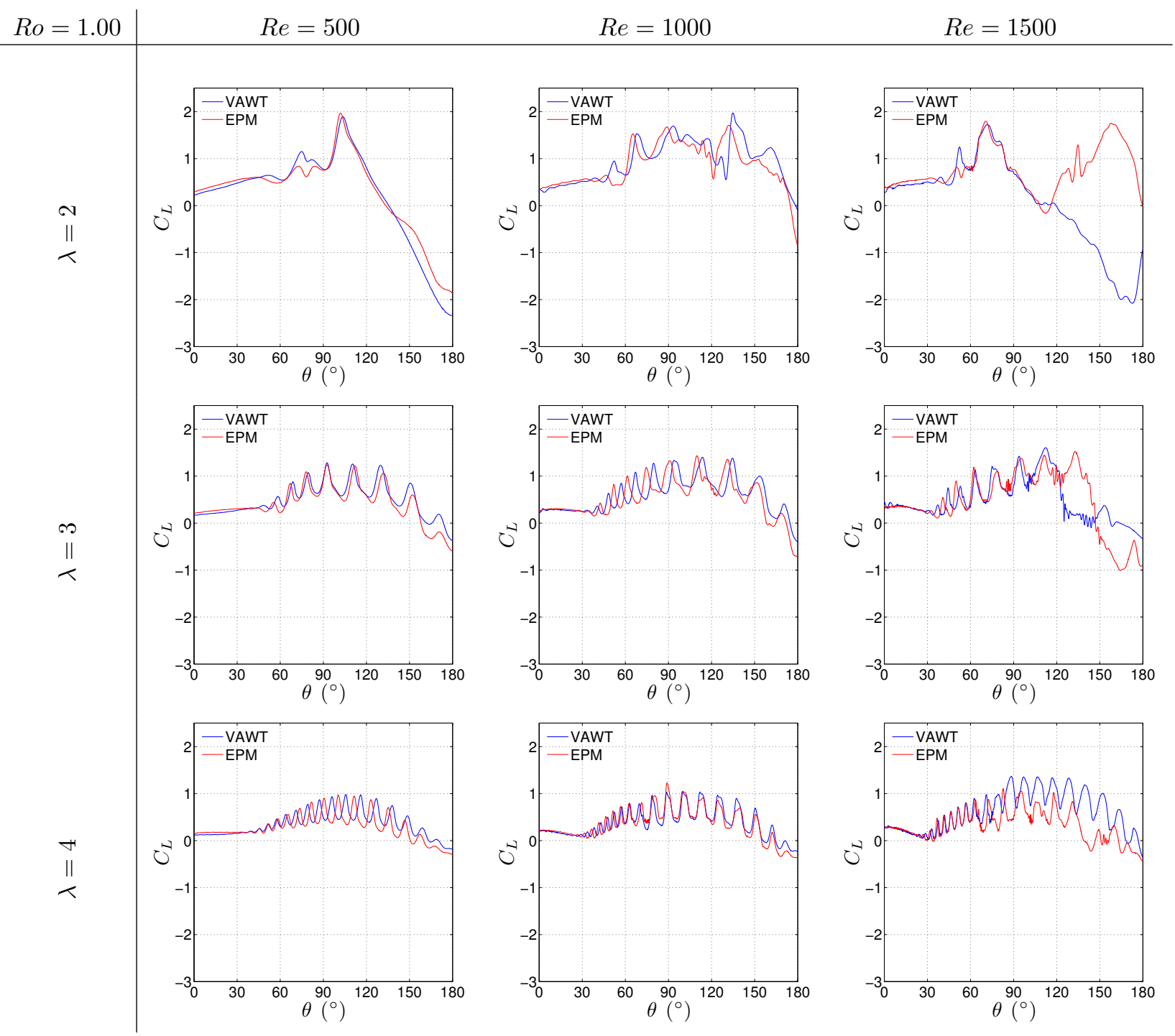

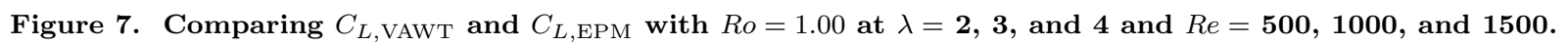




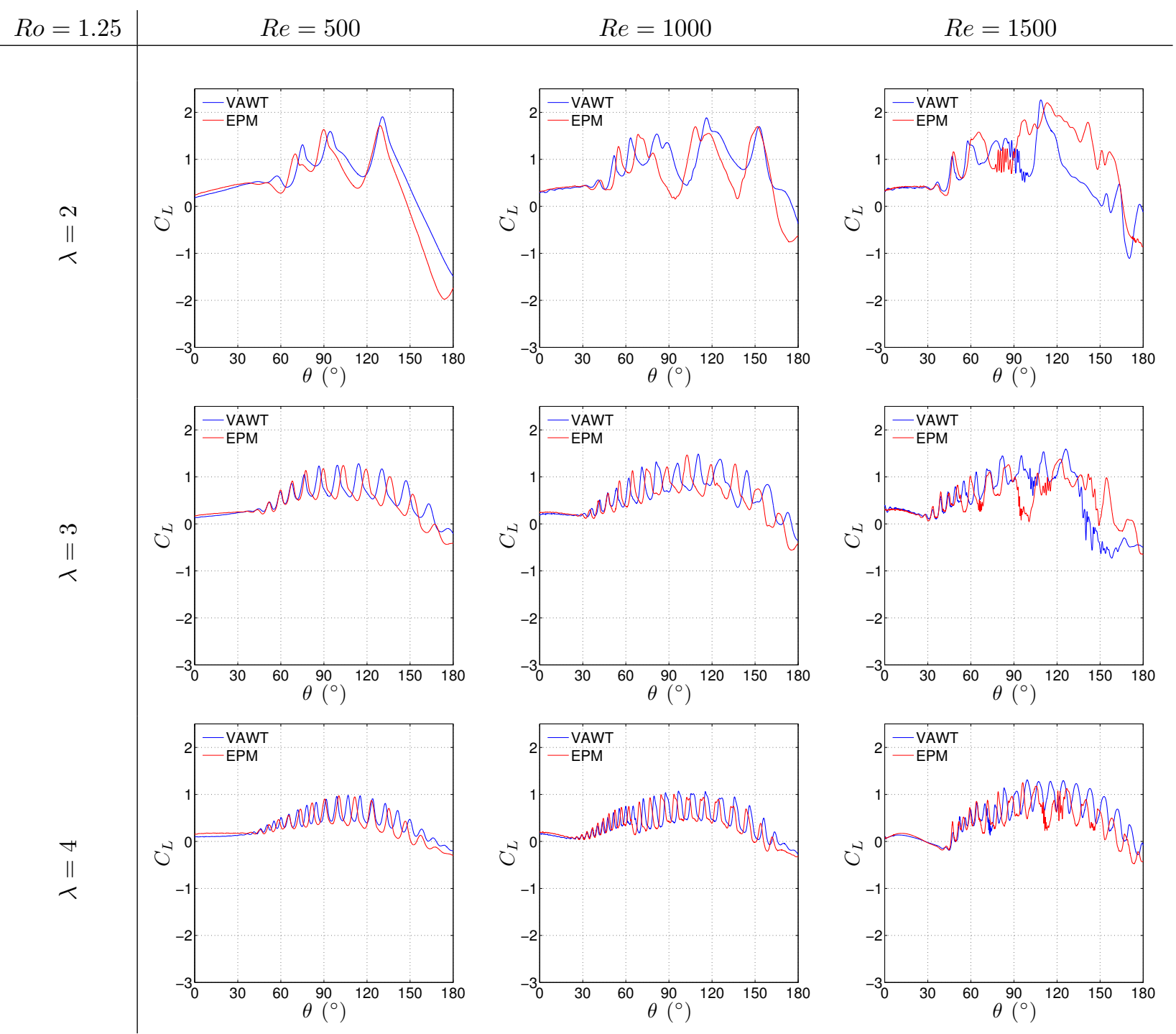

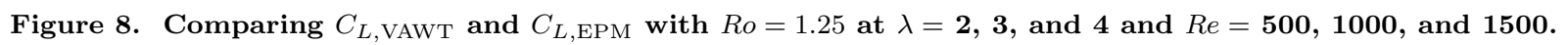




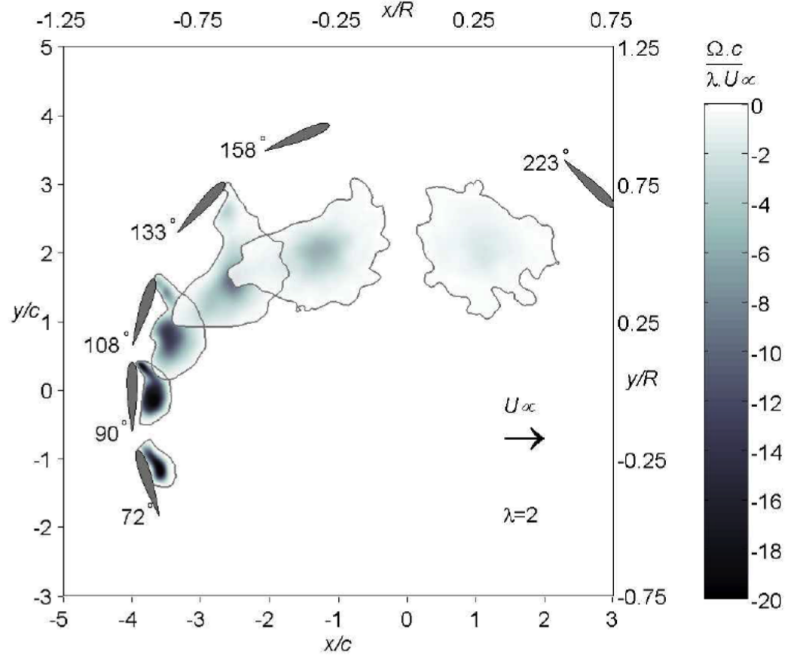

(a) Phase-averaged PIV data of the evolution of LEV reproduced from Ferreira et al.

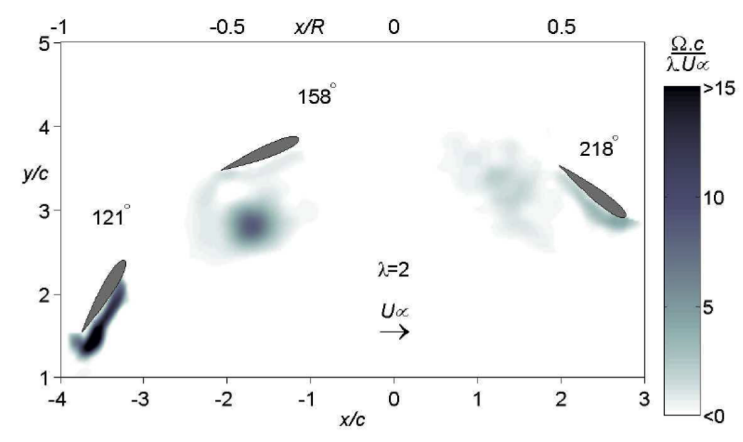

(c) Phase-averaged PIV data of the evolution of TEV reproduced from Ferreira et al.

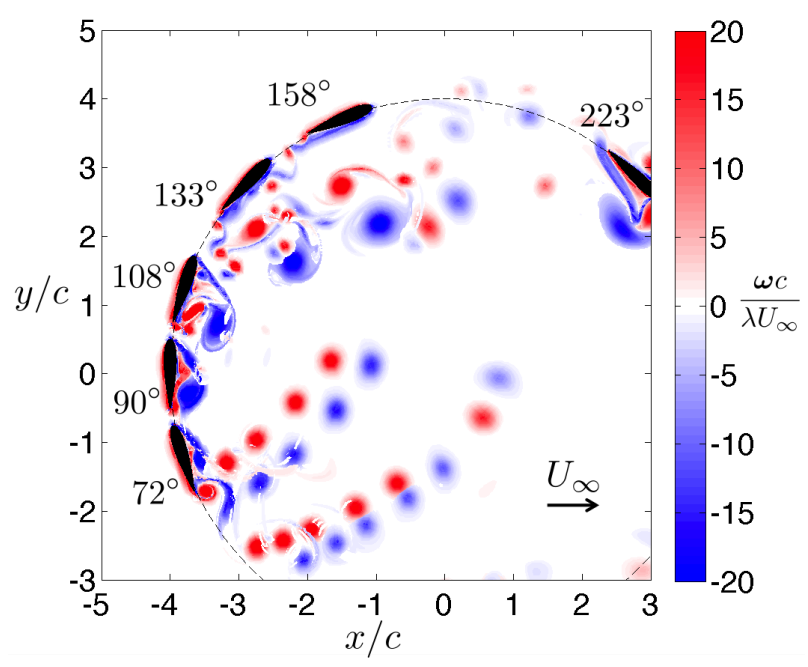

(b) Evolution of vortices in VAWT simulations at azimuthal angles corresponding to figure $9(\mathrm{a})$.

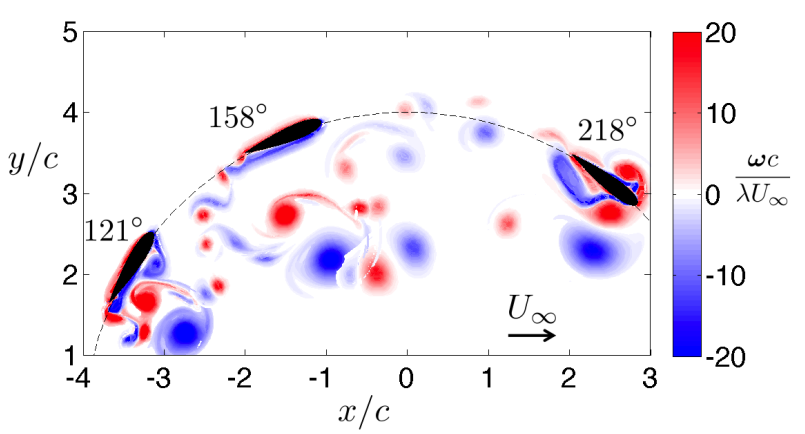

(d) Evolution of vortices in VAWT simulations at azimuthal angles corresponding to figure $9(\mathrm{c})$.

Figure 9. The superposition of the vorticity fields of phase-averaged PIV data from Ferreira et al. $\left(\right.$ Re $\left.\approx 10^{5}\right)$, and of VAWT simulations $(R e=1500)$ at $\lambda=2$ and $R o=1(\ell=4)$ at various azimuthal angles.

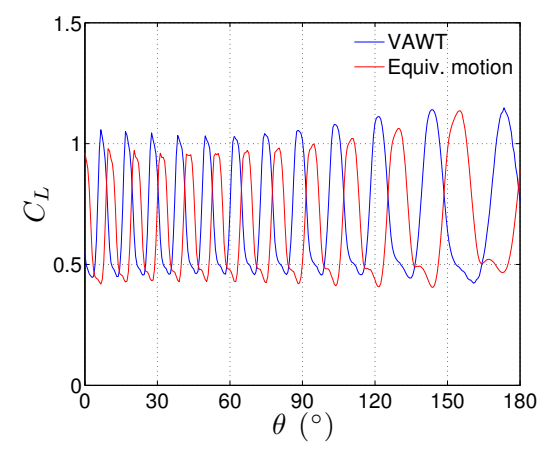

(a) $\alpha=15^{\circ}$

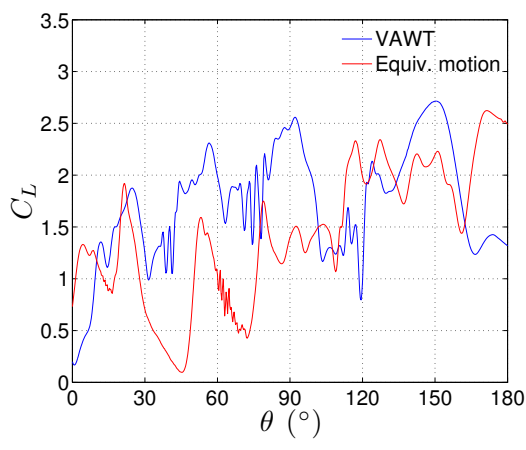

(b) $\alpha=30^{\circ}$

Figure 10. Comparing lift responses of airfoils undergoing only surging motion at $\lambda=2, R o=1.5$, and $R e=1500$. 


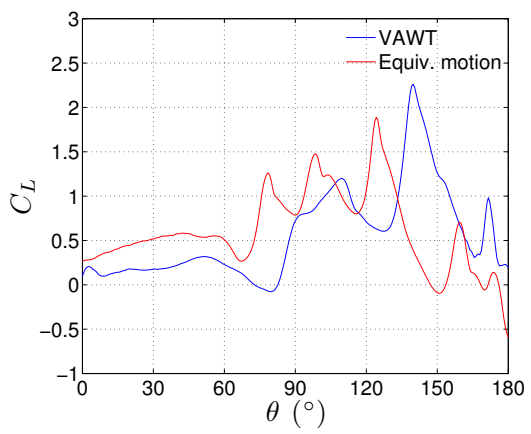

Figure 11. Comparing lift responses of airfoils undergoing only pitching motion at $\lambda=2, R o=1.5$, and $R e=1500$.

Table 1. Observation of the wake-capturing phenomenon for all combinations of the motions.

\begin{tabular}{|c|c|c|c|}
\hline Surging & Pitching & Rotation & Wake-capturing \\
\hline$\bigcirc$ & & & Not observed \\
\hline & $\bigcirc$ & & Not observed \\
\hline & & $\bigcirc$ & Not observed \\
\hline$\bigcirc$ & & $\bigcirc$ & Not observed \\
\hline & $\bigcirc$ & $\bigcirc$ & Not observed \\
\hline$\bigcirc$ & $\bigcirc$ & & Not observed \\
\hline$\bigcirc$ & $\bigcirc$ & $\bigcirc$ & Observed \\
\hline
\end{tabular}

\section{E. Comparison with an airfoil undergoing a sinusoidal motion}

Flows around an airfoil undergoing SPM and SSPM introduced in section II are compared with one undergoing EPM and in VAWT. Let $C_{L, S P M}$ be the lift coefficient of an airfoil undergoing the sinusoidal pitching motion and $C_{L \text {,SSPM }}$ be the lift coefficient of an airfoil undergoing the sinusoidal suring-pitching motion. The comparison of lift responses at $\lambda=2, R o=1.5$, and $R e=1000$ are shown in figure 12 .

In the upstroke phase, we can see that only $C_{L, \mathrm{EPM}}$ is close to $C_{L, \mathrm{VAWT}}$ at low angle of attack. $C_{L, \mathrm{SPM}}$ and $C_{L, \mathrm{SSPM}}$ overestimated the lift. As the angle of attack increases, and after vortex shedding starts, differences between four lift coefficients are relatively small. In the downstroke phase, none of $C_{L, \mathrm{EPM}}, C_{L, \mathrm{SPM}}$, and $C_{L, \mathrm{SSPM}}$ matches the behavior of $C_{L, \mathrm{VAWT}}$. Therefore, sinusoidal motion can not fully catch the dynamics of a rotating airfoil in a VAWT at $\lambda=2$, at least for the sub-scale Reynolds numbers considered in this study.

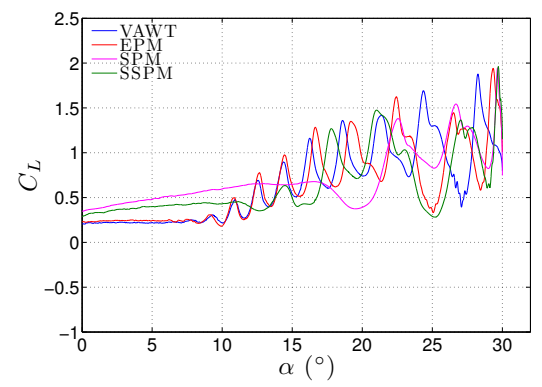

(a) Upstroke phase

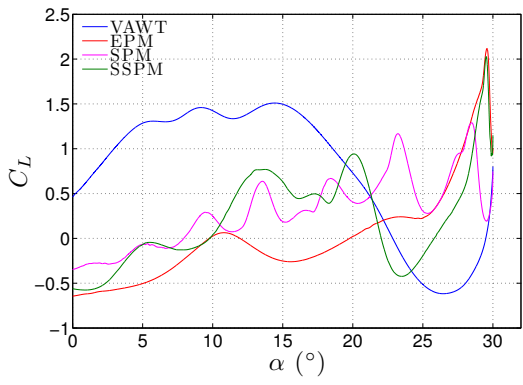

(b) Downstroke phase

Figure 12. Lift coefficients of VAWT, EPM, SPM, and SSPM over a cycle at $\lambda=2, R o=1.5$, and $R e=1000$. 


\section{F. Open loop control on the wake-capturing phenomenon}

From the discussion in sections IIIB and IIIC, the wake-capturing of vortex pair leads to a large decrease in lift. In order to improve the corresponding implied loss in power production, we apply a open loop control on dynamic stall in a VAWT. Inspired by Greenblatt et al., ${ }^{19}$ an actuator modeled as a bodyforce introduced by Joe et $a l .{ }^{24}$ is placed on the suction surface blowing the fluid tangent to the surface to control dynamic stall vortices.

$C_{\mu}$ is the momentum coefficient that defines the momentum injection added by the forcing. For an actuator with the width estimated as the grid spacing, $\Delta x$, which injects a jet with average velocity, $\bar{u}_{\text {jet }}$, the momentum coefficient is

$$
C_{\mu}=\frac{\bar{u}_{\mathrm{jet}}^{2} \Delta x}{\frac{1}{2} U_{\infty}^{2} c}
$$

A bodyforce with strength $C_{\mu}=0.01,0.02,0.04$, and 0.08 is placed at $10 \%, 20 \%$, and $30 \%$ of the chord length from the leading edge to control the baseline flow with wake-capturing phenomenon at $\lambda=2, R o=1.5$, and $R e=1500$. The corresponding lift coefficients are shown in figure 13 .

Since the wake-capturing phenomenon only occurs in the downstroke phase, the control initiates at an azimuthal angle of $90^{\circ}$ and terminates at $180^{\circ}$. We can see that the best improvement is obtained with the actuator placing at $30 \%$ of the chord length from the leading edge. If we average the lift generation in the upwind half of a cycle, the average lift has been increased by $13 \%, 71 \%, 82 \%$, and $94 \%$ with $C_{\mu}=0.01,0.02$, 0.04 , and 0.08 , respectively. Based on these preliminary simulations, with $C_{\mu}$ above a certain threshold, say 0.02 in this case, the control is able to remove the wake-capturing of vortex pair and increase the lift generation significantly in the downstroke phase. Figure 14 shows the vorticity field of the baseline flow and the controlled flow with an actuator placing at $30 \%$ of the chord length from the leading edge with $C_{\mu}=0.02$. We can see that the actuation successfully removes the wake-capturing of vortex pair in the downstroke phase.

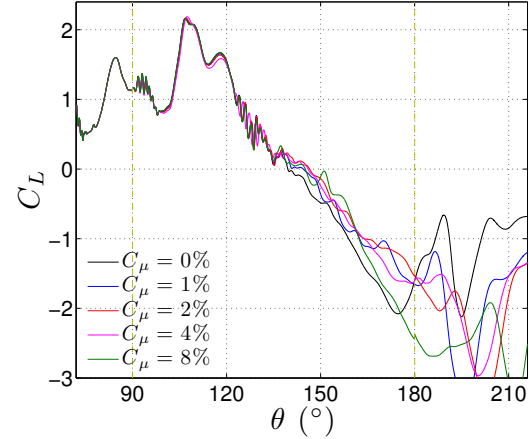

(a) Actuator placed at $10 \% c$

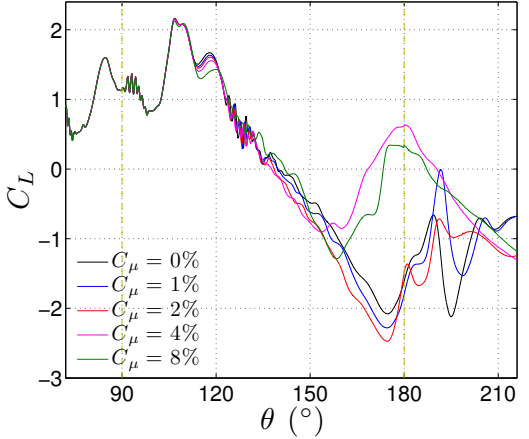

(b) Actuator placed at $20 \% c$

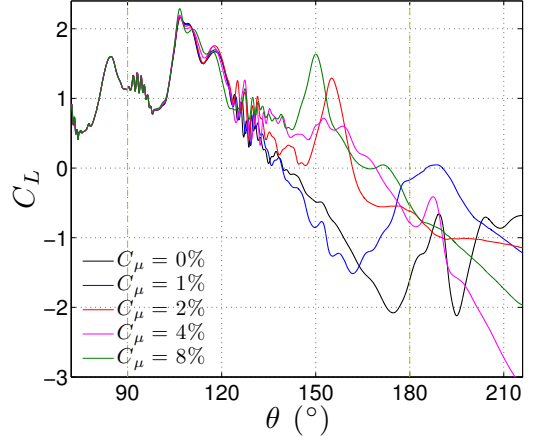

(c) Actuator placed at $30 \% c$

Figure 13. Open loop control on wake-capturing phenomenon with actuator placing at $10 \%, 20 \%$, and $30 \%$ of the chord length from the leading edge at $\lambda=2, R o=1.5$, and $R e=1500$.

\section{Conclusions}

In simulating the flow around a rotating airfoil in a VAWT, we have observed an interesting wakecapturing phenomenon that occurs during the pitch-down portion of the upstream, lift-generating portion of the VAWT cycle. This phenomenon leads to a substantial decrease in lift coefficient due to the presence of a vortex pair traveling together with the rotating airfoil. Our results show that this flow feature persists and grows stronger as tip-speed ratio is reduced and Reynolds number is increased. However, as our study is restricted to two dimensional flow at relatively low Reynolds number, it remains an open question as to whether this feature persists in real application. 

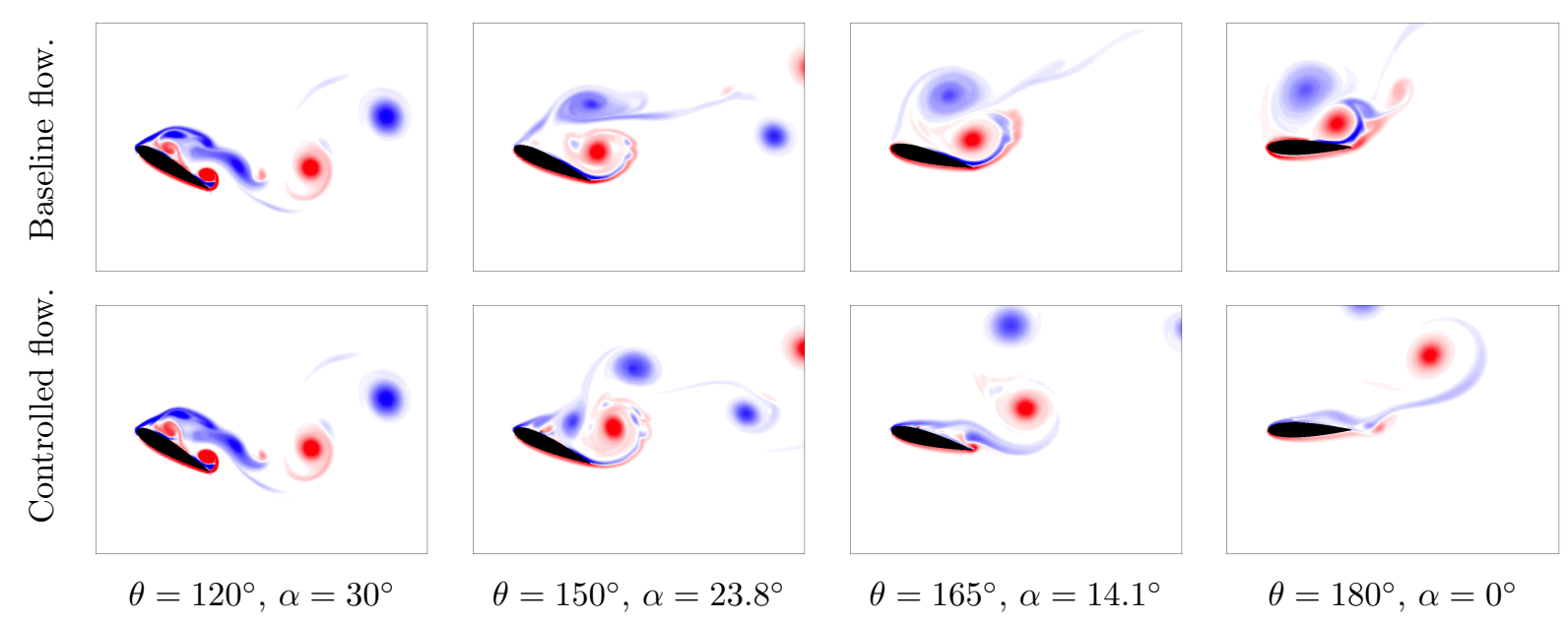

Figure 14. Vorticity field of the baseline flow and the controlled flow with an actuator placing at $30 \%$ of the chord length from the leading edge with $C_{\mu}=0.02$.

An equivalent planar surging-pitching motion was introduced in order to isolate the Coriolis effect on dynamic stall in a VAWT. The results show that the equivalent motion is a good approximation to a rotating airfoil in a VAWT in the upstroke phase where the Coriolis force has relatively small effect on vortices. However, it overestimates the average lift coefficient in the downstroke phase by eliminating the aforementioned wake-capturing.

We further investigated the flow by decomposing the planar motion into surging- and pitching-only motions, where we also compared periodic angle-of-attack and surging velocities corresponding to the real motion, as well as purely sinusoidal variations. We only observe the wake capturing when the combination of surging, pitching, and Coriolis force are present. While the equivalent planar motion captures well the pitch up part of the cycle, all the motions show significant differences in forces during the pitch down motion.

In order to improve the efficiency of a VAWT, an actuator modeled as a bodyforce was used to examine open-loop control in an attempt to suppress the wake-capturing. It is shown that with the actuator placed at appropriate location on the suction surface and the momentum coefficient above a certain threshold, the wake-capturing phenomenon disappears and lift increases significantly in the downstroke phase, with implied increases in power production and efficiency of a VAWT.

In future studies, our numerical results will be compared with the experimental data from McKeon group at Caltech, which will give us more understanding of the aerodynamics in a VAWT. Persistence of the wakecapturing phenomenon in three-dimensional flow at higher Reynolds number also awaits future investigation. Ultimately, we would like to investigate the interactions of multiple VAWTs.

\section{Acknowledgments}

This project is sponsored by the Caltech Field Laboratory for Optimized Wind Energy with Prof. John Dabiri as PI under the support of the Gordon and Betty Moore Foundation. We would like to thank Profs. John Dabiri and Beverley McKeon and Mr. Reeve Dunne for their helpful comments on our work.

\section{Reference}

\footnotetext{
${ }^{1}$ Mertens, S., van Kuik, G., and van Bussel, G., "Performance of an H-Darrieus in the skewed flow on a roof," TransactionsAmerican Society of Mechanical Engineers Journal of Solar Energy Engineering, Vol. 125, No. 4, 2003, pp. 433-440.

${ }^{2}$ Ferreira, C. S., van Bussel, G. J., and van Kuik, G. A., "Wind tunnel hotwire measurements, flow visualization and thrust measurement of a VAWT in skew," Transactions-American Society of Mechanical Engineers Journal of Solar Energy Engineering, Vol. 128, No. 4, 2006, pp. 487.

${ }^{3}$ Dabiri, J. O., "Potential order-of-magnitude enhancement of wind farm power density via counter-rotating vertical-axis
} 
wind turbine arrays," Journal of Renewable and Sustainable Energy, Vol. 3, 2011, pp. 043104.

${ }^{4}$ Kinzel, M., Mulligan, Q., and Dabiri, J. O., "Energy exchange in an array of vertical-axis wind turbines," Journal of Turbulence, Vol. 13, No. 1, 2012.

${ }^{5}$ Ferreira, C. S., van Kuik, G., van Bussel, G., and Scarano, F., "Visualization by PIV of dynamic stall on a vertical axis wind turbine," Experiments in Fluids, Vol. 46, No. 1, 2009, pp. 97-108.

${ }^{6}$ Leishman, J., "Dynamic stall experiments on the NACA 23012 aerofoil," Experiments in fluids, Vol. 9, No. 1-2, 1990, pp. $49-58$.

${ }^{7}$ Raffel, M., Kompenhans, J., and Wernert, P., "Investigation of the unsteady flow velocity field above an airfoil pitching under deep dynamic stall conditions," Experiments in Fluids, Vol. 19, No. 2, 1995, pp. 103-111.

${ }^{8}$ Wang, S., Ingham, D. B., Ma, L., Pourkashanian, M., and Tao, Z., "Numerical investigations on dynamic stall of low Reynolds number flow around oscillating airfoils," Computers \& Fluids, Vol. 39, No. 9, 2010, pp. 1529-1541.

${ }^{9}$ Paraschivoiu, I. and Allet, A., "Aerodynamic analysis of the Darrieus wind turbines including dynamic-stall effects," Journal of Propulsion and Power, Vol. 4, No. 5, 1988, pp. 472-477.

${ }^{10}$ Allet, A., Hallé, S., and Paraschivoiu, I., "Numerical simulation of dynamic stall around an airfoil in Darrieus motion," Journal of Solar Energy Engineering, Vol. 121, No. 1, 1999, pp. 69-76.

${ }^{11}$ Paraschivoiu, I. and Beguier, C., "Visualization, measurements and calculations of dynamic stall for a similar motion of VAWT," Proceedings of the European Wind Energy Conference, Herning, Denmark, 1998.

${ }^{12}$ Hansen, M. and Sresen, D., "CFD model for Vertical Axis Wind Turbine," Wind Energy for the New MillenniumProceedings of the European Wind Energy Conference, Copenhagen, Denmark, 2001.

${ }^{13}$ Nobile, R., Vahdati, M., Barlow, J., and Mewburn-Crook, A., "Dynamic stall for a Vertical Axis Wind Turbine in a two-dimensional study," Wind Energy Applications, Vol. 15, 2011, pp. 4225-4232.

${ }^{14}$ Ferreira, C. S., van Bussel, G., and Van Kuik, G., "2D CFD simulation of dynamic stall on a Vertical Axis Wind Turbine: verification and validation with PIV measurements," 45th AIAA Aerospace Sciences Meeting and Exhibit/ASME Wind Energy Symposium, 2007.

${ }^{15}$ McCroskey, W., Carr, L., and McAlister, K., "Dynamic stall experiments on oscillating airfoils," AIAA Journal, Vol. 14, No. 1, 1976, pp. 57-63.

${ }^{16}$ Wernert, P., Geissler, W., Raffel, M., and Kompenhans, J., "Experimental and numerical investigations of dynamic stall on a pitching airfoil," AIAA journal, Vol. 34, No. 5, 1996, pp. 982-989.

${ }^{17}$ Lee, T. and Gerontakos, P., "Investigation of flow over an oscillating airfoil," Journal of Fluid Mechanics, Vol. 512, 2004, pp. 313-341.

${ }^{18}$ Hwang, I. S., Min, S. Y., Jeong, I. O., Lee, Y. H., and Kim, S. J., "Efficiency improvement of a new vertical axis wind turbine by individual active control of blade motion," Smart Structures and Materials, International Society for Optics and Photonics, 2006, pp. 617311-617311.

${ }^{19}$ Greenblatt, D., Harav, A. B., and Mueller-Vahl, H., "Mechanism of Dynamic Stall Control on a Vertical Axis Wind Turbine," AIAA paper 2013-851, 2013.

${ }^{20}$ Colonius, T. and Taira, K., "A fast immersed boundary method using a nullspace approach and multi-domain far-field boundary conditions," Computer Methods in Applied Mechanics and Engineering, Vol. 197, No. 25, 2008, pp. 2131-2146.

${ }^{21}$ Bloor, M. S., "The transition to turbulence in the wake of a circular cylinder," Journal of Fluid Mechanics, Vol. 19, No. 02, 1964, pp. 290-304.

${ }^{22}$ Dickinson, M. H., Lehmann, F.-O., and Sane, S. P., "Wing rotation and the aerodynamic basis of insect flight," Science, Vol. 284, No. 5422, 1999, pp. 1954-1960.

${ }^{23}$ Choi, J., Colonius, T., and Williams, D., "Dynamics and Energy Extraction of a Surging and Plunging Airfoil at Low Reynolds Number," AIAA paper 2013-672, 2013.

${ }^{24}$ Joe, W. T., Colonius, T., and MacMynowski, D. G., "Feedback control of vortex shedding from an inclined flat plate," Theoretical and Computational Fluid Dynamics, Vol. 25, No. 1-4, 2011, pp. 221-232. 